\title{
Multi-responsive red solid emitter: Detection of trace water and sense of relative humidity
}

\author{
Yuqi Cao ${ }^{1,2}$, Ligong Chen ${ }^{1,2,3^{*}}$, Dongqi Liu ${ }^{1,2}$ and Bowei Wang ${ }^{1,2,3^{*}}$
}

\begin{abstract}
A multi-responsive D-A type compound (CYQ) based on pyrone and triphenylamine was designed and successfully synthesized. The target compound exhibited distinct aggregation-enhanced emission (AEE) effect. Solvatochromic experiment and density functional theory (DFT) indicated CYQ possessed excellent intramolecular charge transfer (ICT) ability. Besides, its mechanofluorochromic property (MFC) was found with a $37 \mathrm{~nm}$ redshift. Powder wide-angle X-ray diffraction (PXRD) and differential scanning calorimetry (DSC) measurements were performed to demonstrate the transformation from the crystalline to amorphous states upon grinding. Surprisingly, CYQ displayed a hypersensitive response to trace water in organic solvents with an excellent detection limit as low as $\mathbf{0 . 0 0 9 6 \%}$ in tetrahydrofuran (THF). Furthermore, it was found that the fluorescent intensity of CYQ declined progressively upon humidity rise, and its color change can be witnessed by naked eyes. Therefore, the relative humidity (RH) sensing strategy guarantees the AIEgen to become a colorimetric sensor under various conditions.
\end{abstract}

Keywords: aggregation-enhanced emission, mechanofluorochromic, trace water, humidity

\section{INTRODUCTION}

With their wide applications in bio-sensors [1], OLED devices [2], $\mathrm{pH}$ indicators [3] and pressure sensors [4], designing and synthesizing organic fluorescent probes in response to unique external stimulation has been a hot spot. However, most of these fluorophores suffer from an "aggregation caused quenching" (ACQ) effect, which severely inhibits their applications in practical situations. Excitingly in 2001, aggregation induced emission (AIE) phenomenon was discovered as a remedy to overcome the above question [5]. Then, numerous templates based on AIE enrich the family of smart materials [6-8]. Among them, red-emitting fluorophores are highly desirable for their distinctive applications in chem- and biosensors [9].

Water plays an important role in many water/oxygen free industrial processes. For example, it is detrimental to synthesizing Grignard reagent in the presence of water, only because trace amount of water can reduce the yield and even quench the reaction. Conventional Karl Fischer method used for water detection usually exhibits some drawbacks, such as time-consuming procedure, costly instrumentation and instability [10]. At this point, AIE compounds were reported as alternatives in water-sensing programs [11]. However, trace water detecting strategies among AIEgens are extremely limited by their poor sensitivity. Therefore, developing an AIE probe with high sensitivity to trace water is urgently desired.

As we know, fluorophores based on D-A skeleton are characterized by their explicit intramolecular charge transfer (ICT) characteristic and strong response to different polarity. Under this circumstance, water affects fluorescent behaviors owing to its high polarity. Herein, our red-emitting compound bearing intensive D-A architecture was conceived as a promising candidate for water detection. This multi-responsive compound displayed aggregation-enhanced emission (AEE) effect accompanied with excellent solvatochromic and mechanofluorochromic (MFC) properties. Moreover, trace water detecting experiment was implemented in three water-miscible organic solvents with an excellent detection limit as low as $0.0096 \%$ in tetrahydrofuran (THF). Besides, relative humidity $(\mathrm{RH})$ sensing strategy ensures that the AIEgen can be a colorimetric sensor under various conditions.

\footnotetext{
${ }^{1}$ School of Chemical Engineering and Technology, Tianjin University, Tianjin 300350, China

${ }^{2}$ Collaborative Innovation Center of Chemical Science and Engineering (Tianjin), Tianjin 300072, China

${ }^{3}$ Tianjin Engineering Research Center of Functional Fine Chemicals, Tianjin 300350, China

* Corresponding authors (emails: lgchen@tju.edu.cn (Chen L); bwwang@tju.edu.cn (Wang B))
} 


\section{EXPERIMENTAL SECTION}

\section{Materials and characterization}

${ }^{1} \mathrm{H}$ NMR and ${ }^{13} \mathrm{C}$ NMR were performed by Bruker Avance $600 \mathrm{MHz}$ spectrometer. High resolution mass spectrum was recorded on a Bruker Paltonicsmicro TOFQ II instrument. Infrared radiation (IR) data were collected on a Nicolet 380FT-IR spectrometer. UV-vis spectra were implemented on Evolution 300 UV-vis spectrophotometer. Liquid and solid photoluminescence (PL) spectra were operated on Hitachi F-2500 spectrophotometer and Horiba Jobin Yvon Fluorolog-3 spectrophotometer, respectively. Emission quantum yields $\left(\Phi_{\mathrm{f}}\right)$ were determined by a FluoroMax-4 (Horiba Jobin Yvon) fluorometer equipped with an integrated sphere. Powder wide angle X-ray diffraction (PXRD) was obtained on a Miniflex 600 Powder X-ray Diffractometer of Rigaku. Differential scanning calorimetry (DSC) experiments were implemented using a Perkin-Elmerat at a heating rate of $10^{\circ} \mathrm{C} \mathrm{min}^{-1}$. Thermal gravimetric analysis (TGA) was measured on an STA 409PC with a heating

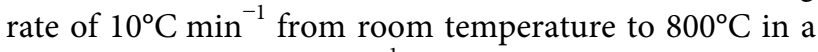
stream of $\mathrm{N}_{2}\left(40 \mathrm{~mL} \mathrm{~min}^{-1}\right)$. All the above experiments were performed at room temperature.

\section{General synthetic procedures}

2-(2,6-dimethyl-4H-pyran-4-ylidene)-1H-indene-1,3(2H)dione (intermediate 1)

To a round bottle flask containing 2,6-dimethyl-4-pyrone $(0.31 \mathrm{~g}, \quad 2.50 \mathrm{mmol})$ and $1 H$-indene-1,3(2H)-dione $(0.44 \mathrm{~g}, 3.00 \mathrm{mmol})$, acetic anhydride $10 \mathrm{~mL}$ was added accordingly. After the mixture was heated under reflux for $6 \mathrm{~h}, \mathrm{pH}$ was adjusted to 7 . The mixture was extracted with ethyl acetate $(3 \times 30 \mathrm{~mL})$ and the organic phase was washed with brine $(5 \times 50 \mathrm{~mL})$, and then concentrated to obtain the crude product. Column chromatography (ethyl acetate/petroleum ether, $1 / 10, v / v)$ was performed to give the yellow intermediate $\mathbf{1}$.

\section{2-(2,6-bis((E)-4-(diphenylamino)styryl)-4H-pyran-4- \\ ylidene)-1H-indene-1,3(2H) dione (CYQ)}

A mixture of intermediate $1(0.63 \mathrm{~g}, 2.50 \mathrm{mmol})$ and 4(diphenylamino)benzaldehyde $(4.1 \mathrm{~g}, 15 \mathrm{mmol})$ was added to $\mathrm{CH}_{3} \mathrm{CN}(15 \mathrm{~mL})$. Piperidine $(3.8 \mathrm{~mL})$ was added subsequently. The mixture was heated under reflux in $\mathrm{N}_{2}$ for $24 \mathrm{~h}$. After cooling to room temperature, the crude product was purified by column chromatography (dichloromethane, DCM) to yield the target compound, red solid (1.18 g), $62 \%$ yield. m.p. $281-283^{\circ} \mathrm{C} .{ }^{1} \mathrm{H}$ NMR
$\left(\mathrm{CDCl}_{3}, 600 \mathrm{MHz}\right) \delta(\mathrm{ppm}): 8.43(\mathrm{~s}, 2 \mathrm{H}), 7.77-7.75(\mathrm{~m}$, $2 \mathrm{H}), 7.61-7.59(\mathrm{~m}, 2 \mathrm{H}), 7.47(\mathrm{~s}, 1 \mathrm{H}), 7.44-7.43(\mathrm{~d}$, $J=8.4 \mathrm{~Hz}, 5 \mathrm{H}), 7.33(\mathrm{~s}, 2 \mathrm{H}), 7.31(\mathrm{~s}, 4 \mathrm{H}), 7.30(\mathrm{~s}, 2 \mathrm{H})$, $7.16-7.15(\mathrm{~d}, J=7.8 \mathrm{~Hz}, 8 \mathrm{H}), 7.12(\mathrm{~s}, 1 \mathrm{H}), 7.11(\mathrm{~s}, 2 \mathrm{H})$, $7.09(\mathrm{~s}, 1 \mathrm{H}), 7.06-7.05(\mathrm{~d}, J=9 \mathrm{~Hz}, 4 \mathrm{H}), 6.77(\mathrm{~s}, 1 \mathrm{H}), 6.76$ (s, $1 \mathrm{H}) .{ }^{13} \mathrm{C}$ NMR $\left(\mathrm{CDCl}_{3}, 100 \mathrm{MHz}\right) \delta(\mathrm{ppm}): 192.78$, $160.61,149.61,148.48,146.90,140.79,136.01,133.07$, $129.53,128.87,128.22,125.43,124.08,121.87,121.25$, 117.57, 108.44. HRMS (ESI): calcd. for $\mathrm{C}_{54} \mathrm{H}_{38} \mathrm{~N}_{2} \mathrm{O}_{3}$ : 763.2882 $(\mathrm{M}+\mathrm{H})^{+}$, found 763.2954. IR $\left(\mathrm{KBr}, \mathrm{cm}^{-1}\right)$ : $3,433,1,691,1,645,1,589,1,494,1,401,1,322,1,278,1,237$, 1,167, 949, 749, 698.

\section{RESULTS AND DISCUSSION}

The target compound CYQ was easily obtained from the condensation of pyrone with intermediate $\mathbf{1}$ [12]. General synthetic route and the structural characterization of CYQ were summarized in the supporting information (Scheme S1, Figs S1-S4). The absorption spectrum of CYQ in DCM is shown in Fig. 1b. It presents two independent peaks. The peak centered at $360 \mathrm{~nm}$ can be attributed to the $\pi-\pi^{*}$ transition. Meanwhile, the one located at $525 \mathrm{~nm}$ was ascribed to the ICT transition due to the strong push-pull effect [13].

The AEE process was confirmed in DCM-hexane system. As we can see in Fig. 1c, in the molecularly dissolved solution (DCM), CYQ emitted weak red fluorescence. In this regard, the molecules exhibit a random torsion pattern, which leads to non-radiative pathway and weak fluorescence [14,15]. However, more distinctive red fluorescence was recorded upon addition of poor solvent (hexane) until reaching the maximum PL intensity at 50\% $n$-hexane fraction $\left(\Phi_{\mathrm{f}}, 33.93 \%\right)$. In this sense, the restriction of intramolecular rotation (RIR) plays a leading role. When hexane was added, the free rotation pathway was limited to some extent. In this case, the non-radiative decay was blocked, giving rise to higher PL intensity [16]. Conversely, the fluorescent intensity began to decline along with the increase of $n$-hexane fraction (Fig. 1d) which may be attributed to the formation of amorphous aggregates [17]. Interestingly, the whole PL profile displayed a hypochromatic tendency. This phenomenon may be ascribed to the repressed ICT process induced by reduced polarity. Moreover, the distorted molecular skeleton aroused by aggregation may also contribute to the blue-shifted spectrum.

Moreover, ICT process was confirmed by solvatochromic experiment. As shown in Fig. 2a, the fluorescent peak of CYQ appears at $566 \mathrm{~nm}$ in cyclohexane and displays a bathochromic tendency along with the in- 
a<smiles>O=C1C(=C2C=C(/C=C/c3ccc(N(c4ccccc4)c4ccccc4)cc3)OC(/C=C/c3ccc(N(c4ccccc4)c4ccccc4)cc3)=C2)C(=O)c2ccccc21</smiles>

CYQ

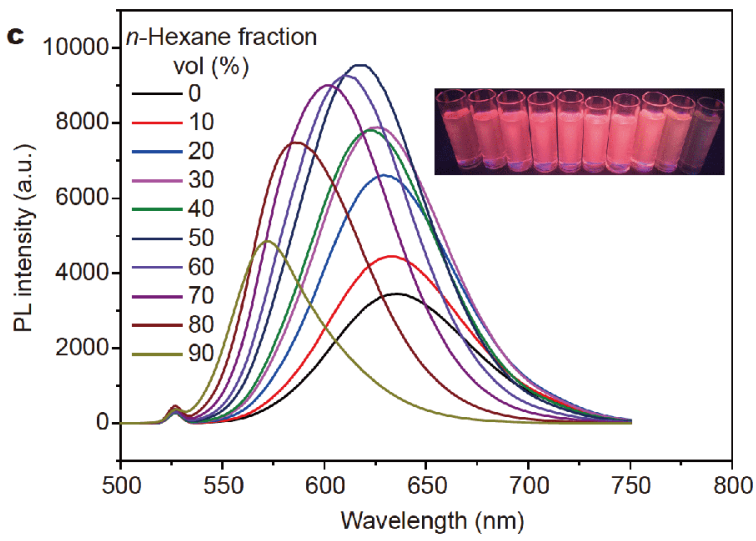

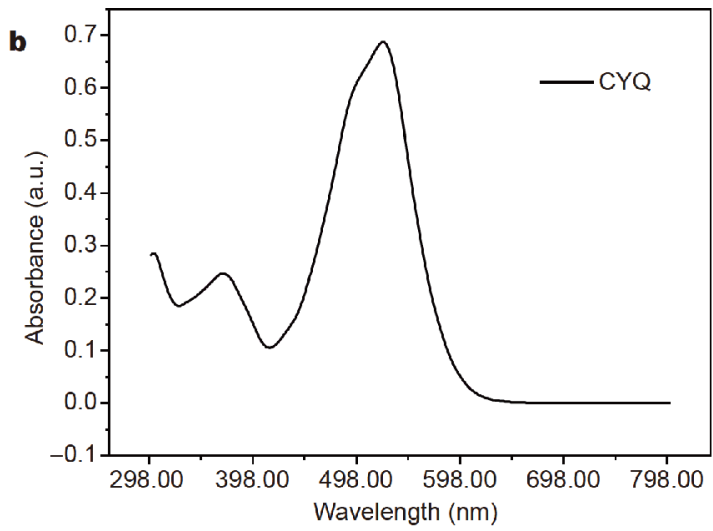

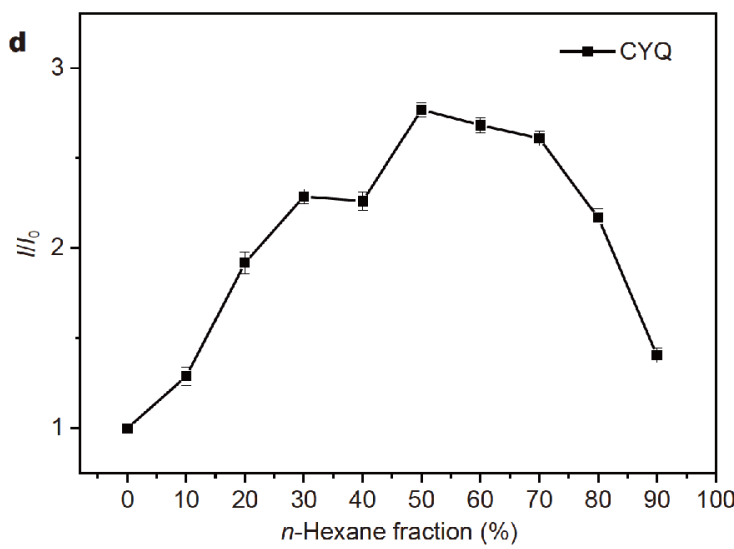

Figure 1 (a) Chemical structure of CYQ; (b) UV-vis absorption spectra of CYQ; (c) PL spectra in DCM-hexane mixtures. The inset depicts the emission image under $365 \mathrm{~nm}$ UV illumination; (d) changes in PL intensity. Error bars were calculated using Origin 8.

creasing of solvent polarity, with up to $100 \mathrm{~nm}$ shift in DMF. According to Guan et al. [18], molecules based on D-A type favor charge separation which can be stabilized by solvation, and trigger the reduction of energy gap between the highest occupied molecular orbital (HOMO) and lowest unoccupied molecular orbital (LUMO). Because of the above driving force, a red-shifted PL spectrum was observed, accompanied with the enhanced polarity. This superb linear color change characteristic (Fig. 2b) makes it possible for CYQ to serve as a promising candidate to distinguish different solvents [19]. In addition, the spatial distributions of HOMO and LUMO were calculated with density functional theory (DFT). As depicted in Fig. 2c, the HOMO of CYQ is exclusively located in the triphenylamine unit while the LUMO orbits are shifted to the pyrone core. This prominent charge separation provides compelling evidence to the above solvatochromic studies. Collectively, an unambiguous ICT transmission through the entire system is anticipated.

The mechanochromic property of CYQ $\left(\Phi_{\mathrm{f}}, 7.85 \%\right)$ was examined in Fig. 3a. A conspicuous red-shifted MFC feature can be witnessed, which displays an alternative fluorescence from red to purplish red. The $37 \mathrm{~nm}$ shift may be attributed to the planar structure upon grinding. Besides, the excellent ICT process may promote the MFC behavior [20].

TGA was carried out to verify the high thermal stability of CYQ, losing $5 \%$ of its weight at $410^{\circ} \mathrm{C}$ (Fig. S5). To obtain a further understanding of the MFC nature, PXRD and DSC measurements were performed. As shown in Fig. $3 b$, the sharp scattering peaks indicate a regular crystalline structure. However, for the pattern of the ground solid, most diffraction peaks remarkably weakened or disappeared, indicating an amorphous state [21]. The thermal property of CYQ was analyzed by DSC. As demonstrated in Fig. 3c, no additional peaks can be detected for the original sample except for the sharp melting peak centered at $281^{\circ} \mathrm{C}$, while for the ground solid, the exothermic transition shoulder at $168^{\circ} \mathrm{C}$ could be ascribed to the cold-crystallization process upon annealing [22]. 

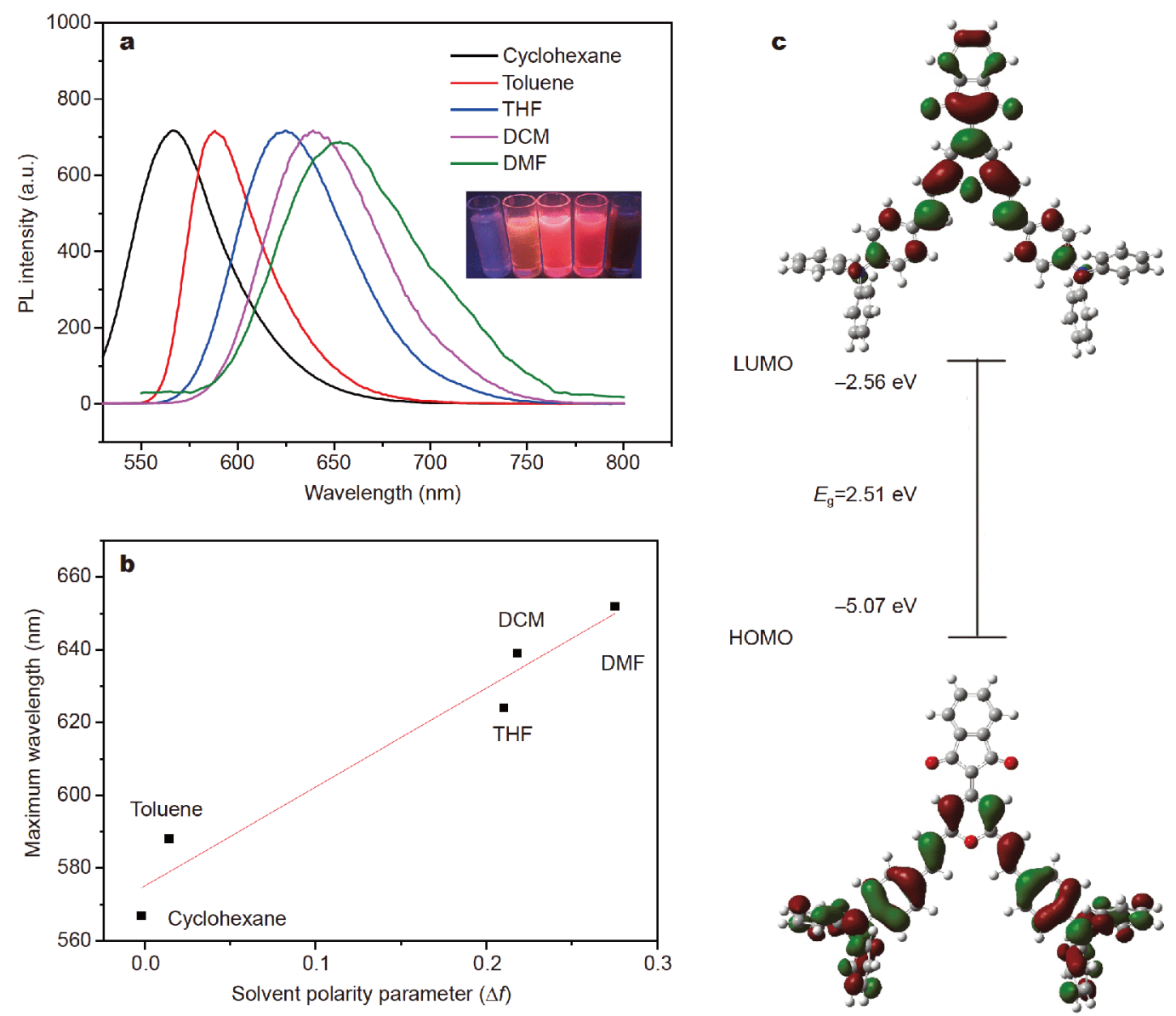

Figure 2 (a) PL spectrum in cyclohexane, toluene, THF, DCM and N,N-dimethylformamide (DMF); (b) plots of maximum wavelength vs. solvent polarity parameter $(\Delta f)$; (c) B3LYP/6-31G(d) calculated molecular orbital amplitude plots and their calculated HOMO-LUMO energy gap (eV).
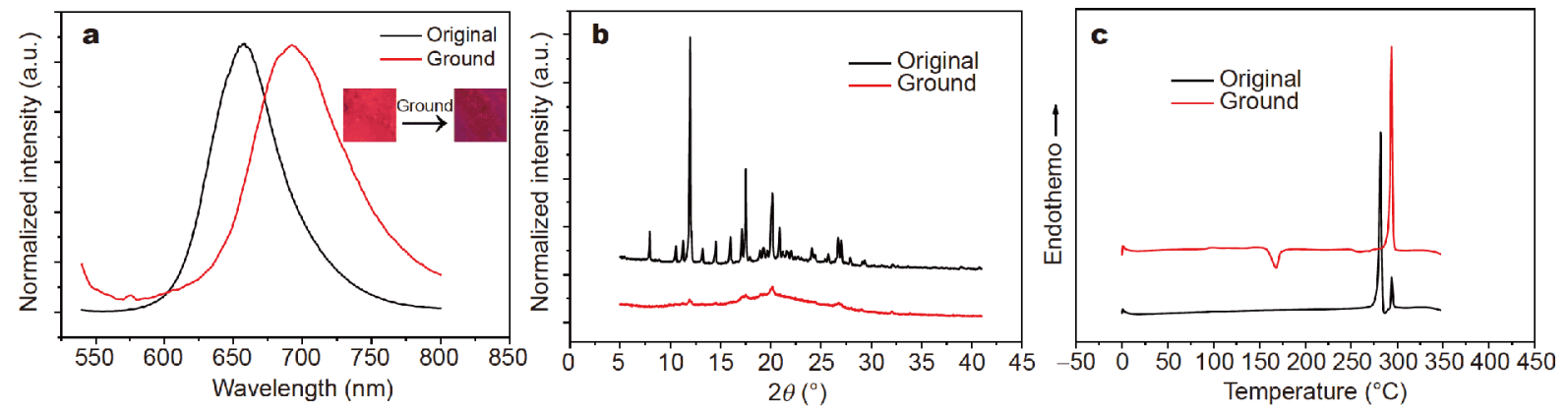

Figure 3 (a) Normalized PL emission spectra of CYQ upon grinding. The insets depict the fluorescence images (original and ground state) taken under $365 \mathrm{~nm}$ UV lamp. (b) PXRD patterns and (c) DSC curves of original and ground states.

On account of the specific D- $\pi$-A skeleton and superb ICT characteristic, it is possible for the target compound to present hypersensitive behavior in response to different polarity. Under this circumstance, three water-miscible organic solvents were chosen as representatives. As shown in Fig. 4a, the fluorescence intensity in $\operatorname{THF}\left(\Phi_{\mathrm{f}}\right.$, 27.13\%) exhibited a descending tendency along with increasing water fraction. A drastic declination to $47 \%$ was 

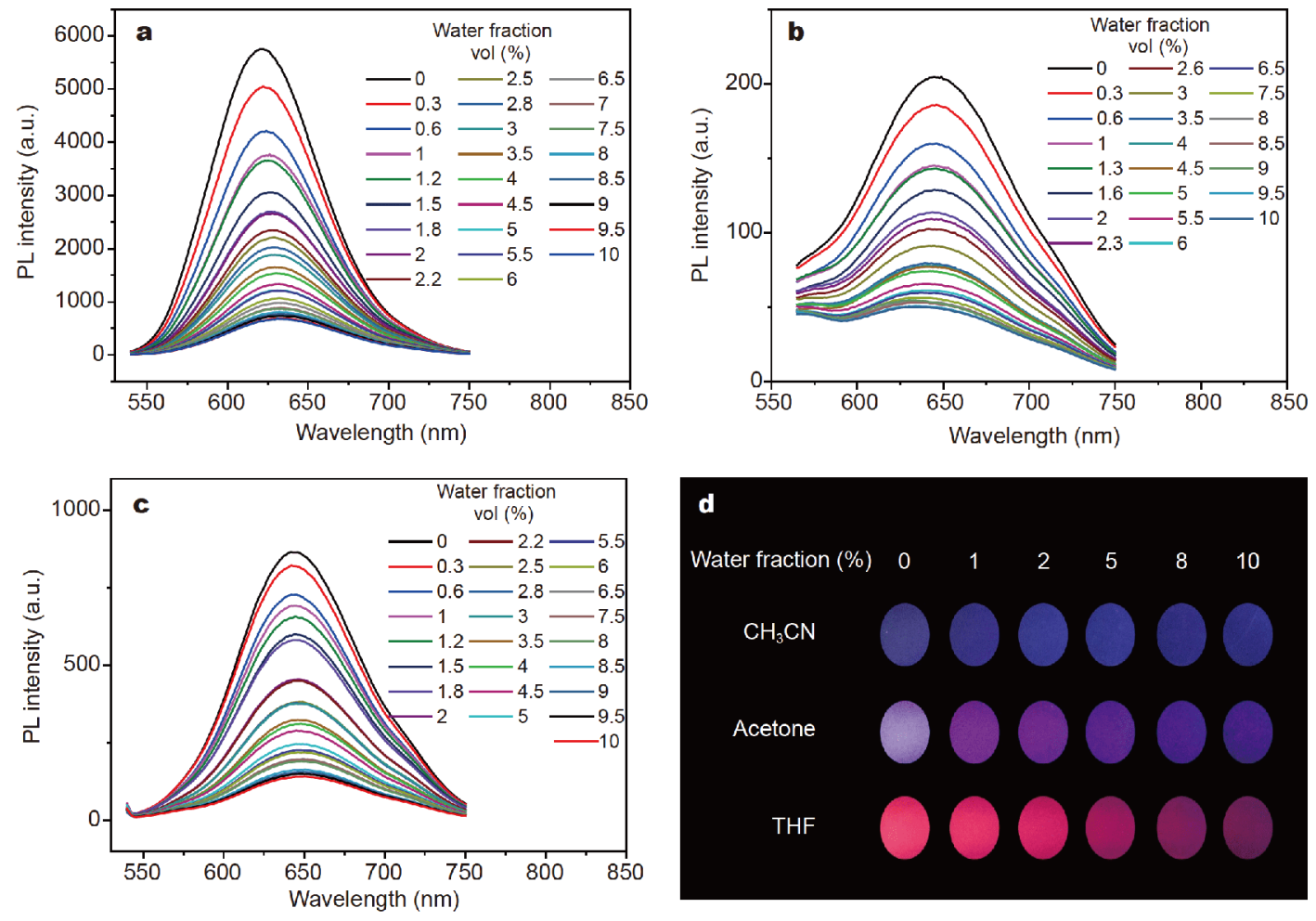

Figure 4 PL spectra of CYQ in (a) THF, (b) acetonitrile, and (c) acetone/water mixtures; (d) emission images in 0\%-10\% water fraction under $365 \mathrm{~nm}$ UV illumination.

displayed when the water fraction was reduced to $1.8 \%$ $(v / v)$, whereas this downtrend became moderate when the water content exceeded $3.0 \%(v / v)$. Meanwhile, the fluorescent intensity of the target compound revealed a linear relationship and issued an excellent detection limit of $0.0096 \%$ (Fig. 5a). Unsurprisingly, trace water detection experiment displayed a similar trend in acetonitrile/ acetone-water system and yielded a detection limit as low as $0.0191 \%$ and $0.0123 \%$, respectively (Fig. 5c, e). Moreover, the fluorescent intensity was performed to show the similar downtrend (Fig. 5b, d, f). In order to demonstrate the effect of trace water, fluorescent images were taken for further discussion. As illustrated in Fig. 4d, a gradual bathochromic emission accompanied with a plunging PL intensity was clearly observed when the water fraction increased from $0 \%$ to $10 \%(v / v)$. All of the above charactersistic pave the way for constructing a highly sensitive sensor for trace water in water-miscible organic solvents.

In addition, the RH sensing strategy was performed at $10 \%, 24 \%, 43 \%, 63 \%$ and $96 \%$ RH content. As we can see in Fig. 6. the fluorescent intensity declined progressively with humidity rise. As humidity increases, water mole- cules are gradually absorbed by the target compound. At this point, ICT process was triggered by the higher ambient polarity, which resulted in lower emission intensity. Interestingly, these calibration lines exert linear relationship corresponding to the relevant $\mathrm{RH}$, indicating a great possibility in serving as a colorimetric sensor under various conditions.

\section{CONCLUSION}

In this work, we outlined a multi-response red-emitting pyrone derivative characterized by AEE property. Moreover, solvatochromic experiment was implemented to examine the ICT process which was manifested by DFT calculation. Besides, our AIEgen exhibited substantial quenching along with fluorescent color change from red to purplish red upon grinding. The mechanism of this $37 \mathrm{~nm}$ MFC feature was demonstrated by XRD and DSC experiments. Moreover, owing to the explicit ICT effect, CYQ was chosen as a trace water sensor in commonly used water-miscible organic solvents. In particular, the detection limit as low as $0.0096 \%$ in THF indicates the practical application in daily lives. Furthermore, the solid state CYQ can also serve as a linear colorimetric sensor 

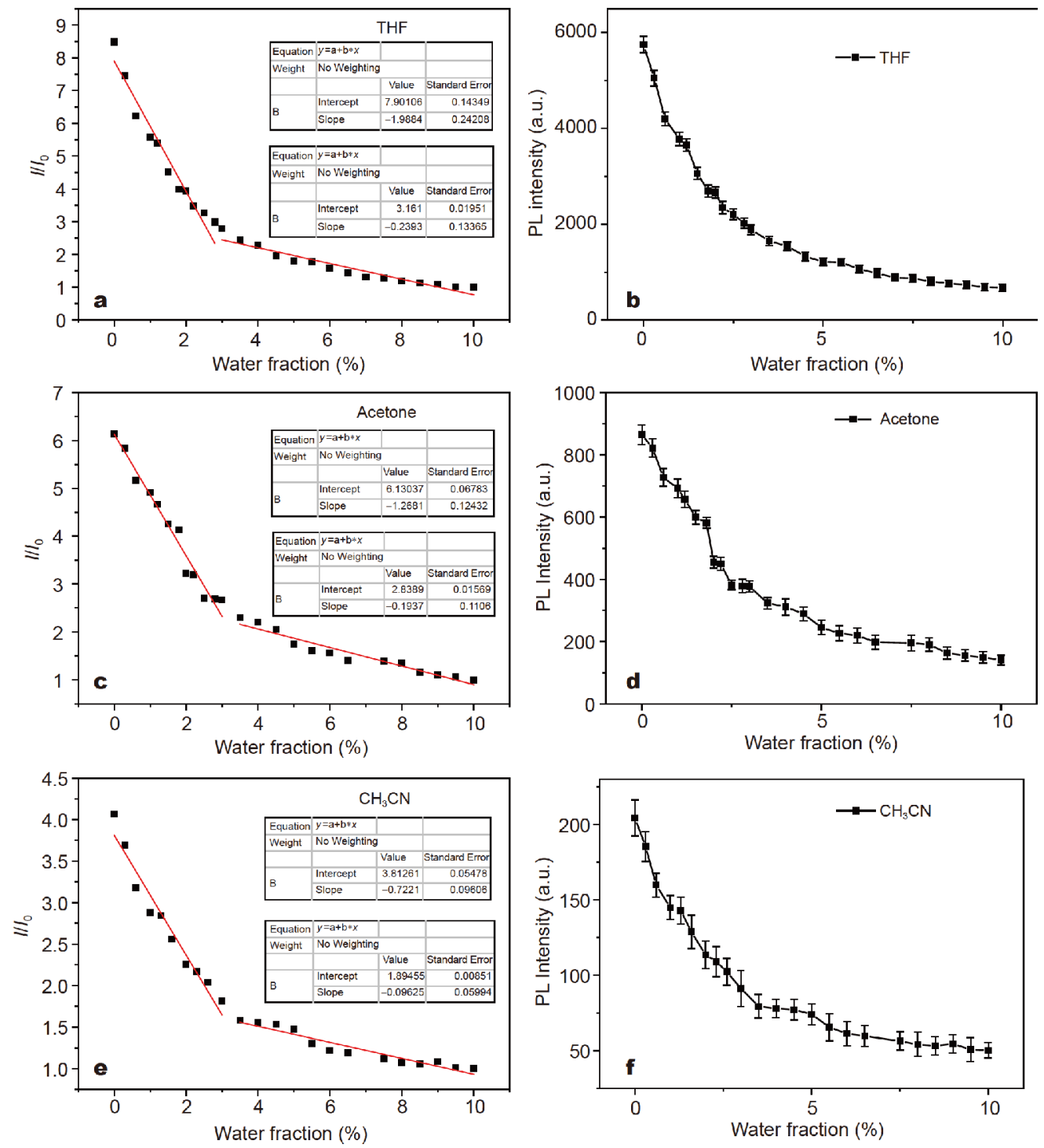

Figure 5 Detection limits in (a) THF, (c) acetone and (e) $\mathrm{CH}_{3} \mathrm{CN}$. PL spectrum of $\mathbf{C Y Q}$ in (b) THF/water, (d) acetone/water and (f) $\mathrm{CH}{ }_{3} \mathrm{CN} /$ water. Error bars were calculated using Origin 8.
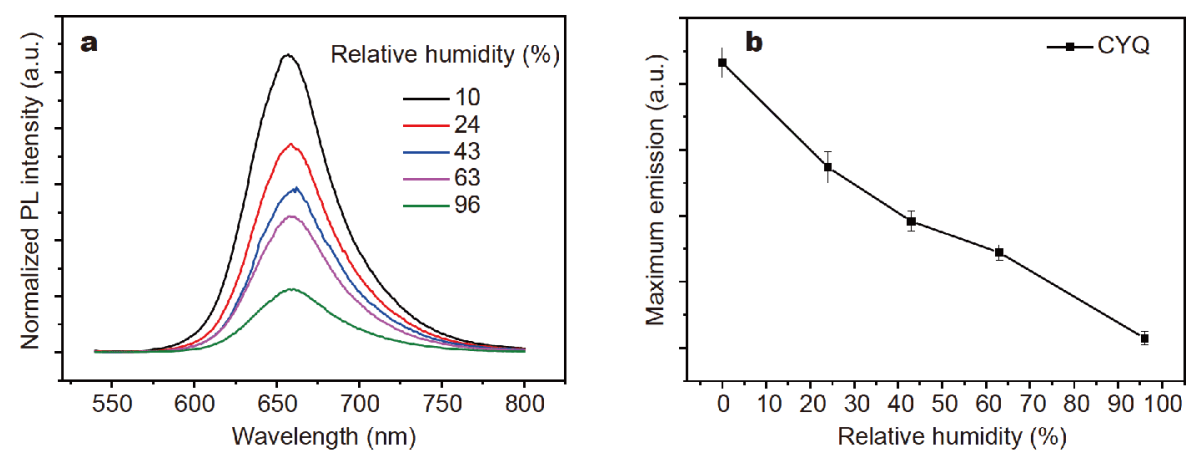

Figure 6 (a) PL emission spectra under different RH conditions; (b) plots of relative humidity versus maximum emission. Error bars were calculated using Origin 8. 
under different RH conditions.

\section{Received 17 September 2018; accepted 26 October 2018; published online 15 November 2018}

1 Liu J, Chen C, Ji S, et al. Long wavelength excitable near-infrared fluorescent nanoparticles with aggregation-induced emission characteristics for image-guided tumor resection. Chem Sci, 2017, 8: $2782-2789$

2 Song $\mathrm{F}, \mathrm{Xu} \mathrm{Z}$, Zhang Q, et al. Highly efficient circularly polarized electroluminescence from aggregation-induced emission luminogens with amplified chirality and delayed fluorescence. Adv Funct Mater, 2018, 28: 1800051-1800063

3 Chen S, Liu J, Liu Y, et al. An AIE-active hemicyanine fluorogen with stimuli-responsive red/blue emission: extending the $\mathrm{pH}$ sensing range by "switch + knob" effect. Chem Sci, 2012, 3: 1804-1809

4 Shi P, Duan Y, Wei W, et al. A turn-on type mechanochromic fluorescent material based on defect-induced emission: implication for pressure sensing and mechanical printing. J Mater Chem C, 2018, 6: 2476-2482

5 Luo J, Xie Z, Lam JWY, et al. Aggregation-induced emission of 1methyl-1,2,3,4,5-pentaphenylsilole. Chem Commun, 2001, 17401741

6 Wang B, Chen P, Zhang J, et al. Self-assembled core-shell-corona multifunctional non-viral vector with AIE property for efficient hepatocyte-targeting gene delivery. Polym Chem, 2017, 8: 74867498

7 Zhu H, Huang J, Kong L, et al. Branched triphenylamine luminophores: Aggregation-induced fluorescence emission, and tunable near-infrared solid-state fluorescence characteristics via external mechanical stimuli. Dyes Pigments, 2018, 151: 140-148

8 Liu D, Li J, Liu J, et al. A new organic compound of 2-(2,2-diphenylethenyl)anthracene (DPEA) showing simultaneous electrical charge transport property and AIE optical characteristics. J Mater Chem C, 2018, 6: 3856-3860

9 Xia Q, Chen Z, Yu Z, et al. Aggregation-induced emission-active near-infrared fluorescent organic nanoparticles for noninvasive long-term monitoring of tumor growth. ACS Appl Mater Interfaces, 2018, 10: 17081-17088

10 Wang D, Zhao $\mathrm{H}, \mathrm{Li} \mathrm{H}$, et al. A fluorescent "glue" of water triggered by hydrogen-bonding cross-linking. J Mater Chem C, 2016, 4: 11050-11054

11 Wang L, Yang L, Li L, et al. The synthesis and highly sensitive detection of water content in THF using a novel solvatochromic AIE polymer containing diketopyrrolopyrrole and triphenylamine. New J Chem, 2016, 40: 6706-6713

12 Liu Y, Lei Y, Li F, et al. Indene-1,3-dionemethylene-4H-pyran derivatives containing alkoxy chains of various lengths: aggregation-induced emission enhancement, mechanofluorochromic properties and solvent-induced emission changes. J Mater Chem C, 2016, 4: $2862-2870$
13 Hancock JM, Gifford AP, Zhu Y, et al. $n$-Type conjugated oligoquinoline and oligoquinoxaline with triphenylamine endgroups: efficient ambipolar light emitters for device applications. Chem Mater, 2006, 18: 4924-4932

14 Lin Y, Chen G, Zhao L, et al. Diethylamino functionalized tetraphenylethenes: structural and electronic modulation of photophysical properties, implication for the CIE mechanism and application to cell imaging. J Mater Chem C, 2015, 3: 112-120

15 Zhao L, Lin Y, Liu T, et al. Rational bridging affording luminogen with AIE features and high field effect mobility. J Mater Chem C, 2015, 3: 4903-4909

16 Venkatramaiah N, Kumar GD, Chandrasekaran Y, et al. Efficient blue and yellow organic light-emitting diodes enabled by aggregation-induced emission. ACS Appl Mater Interfaces, 2018, 10: 3838-3847

17 Cao YQ, Xi Y, Teng XY, et al. Alkoxy substituted D- $\pi$-A dimethyl4-pyrone derivatives: Aggregation induced emission enhancement, mechanochromic and solvatochromic properties. Dyes Pigments, 2017, 137: 75-83

18 Guan XL, Jia TM, Zhang DH, et al. A new solvatochromic linear $\pi$ conjugated dye based on phenylene-(poly)ethynylene as supersensitive low-level water detector in organic solvents. Dyes Pigments, 2017, 136: 873-880

19 Yuan WZ, Gong Y, Chen S, et al. Efficient solid emitters with aggregation-induced emission and intramolecular charge transfer characteristics: molecular design, synthesis, photophysical behaviors, and OLED application. Chem Mater, 2012, 24: 1518-1528

20 Cao YQ, Chen L, Xi Y, et al. Stimuli-responsive 2,6-diarylethene4H-pyran-4-one derivatives: Aggregation induced emission enhancement, mechanochromism and solvatochromism. Mater Lett, 2018, 212: 225-230

21 Gao H, Xu D, Wang Y, et al. Effects of alkyl chain length on aggregation-induced emission, self-assembly and mechanofluorochromism of tetraphenylethene modified multifunctional $\beta$ diketonate boron complexes. Dyes Pigments, 2018, 150: 59-66

$22 \mathrm{Bu} \mathrm{L}, \mathrm{Li}$ Y, Wang J, et al. Synthesis and piezochromic luminescence of aggregation-enhanced emission 9,10-bis(N-alkylcarbazol-2-ylvinyl-2)anthracenes. Dyes Pigments, 2013, 99: 833-838

Acknowledgements We are grateful for the financial support from the National Natural Science Foundation of China (21576194).

Author contributions Cao Y carried out the experiments with assistance of Chen L. All authors discussed the results and commented on the manuscript and Cao Y wrote the manuscript.

Conflict of interest The authors declare no conflict of interest

Supplementary information Supporting data are available in the online version of the paper. 

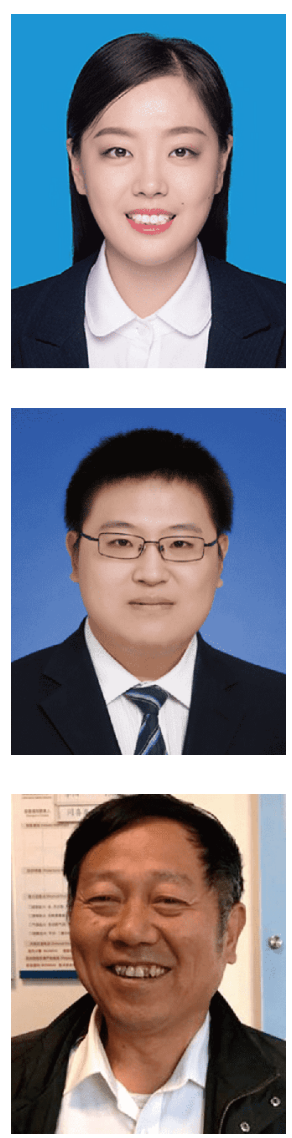

Yuqi Cao is a PhD student at Tianjin University, under the supervision of Prof. Ligong Chen. Currently, she is working on the development of aggregation induced emission mechanofluorochromic materials.

Bowei Wang is a lecturer at the School of Chemical Engineering and Technology, Tianjin University. His current research interest includes catalysis, functional materials, carbon materials, and synthesis of fine chemicals.

Ligong Chen is a professor at Tianjin University. He obtained his PhD degree from University of Nottingham. His research includes aggregation induced emission mechanofluorochromic materials, functional carbon materials, and fluorescence probes.

\section{刺激响应红色发光体: 痕量水及相对湿度的检测}

曹宇奇 ${ }^{1,2}$, 陈立功 ${ }^{1,2,3^{*}}$, 刘东琦 ${ }^{1,2}$, 王博威 ${ }^{1,2,3^{*}}$

摘要 本文设计并开发了一种以吡喃酮和三苯胺为母体的多刺激响应 D-A型化合物(CYQ). 目标产物表现出优异的聚集诱导发光增强性 能(AEE). 溶剂变色实验及密度泛函理论表明CYQ具有极强的分子内电荷转移(ICT)性质. 粉末广角X射线衍射(PXRD)及差式扫描量热法 (DSC) 证明了在外力作用下, 分子由晶态向无定型态的转变. 研究发现, CYQ对有机溶液中的痕量水非常敏感, 在四氢呋喃(THF)中可达到 极低的检出限 $0.0096 \%$. 除此之外, 随着湿度的增加, CYQ的荧光强度呈现逐渐下降趋势. 这一相对湿度检测法为该AIE材料成为不同环境 下的比色探针提供了可能. 